\title{
ON THE STRUCTURE OF QUANTUM AUTOMORPHISM GROUPS
}

\author{
CHRISTIAN VOIGT
}

\begin{abstract}
We compute the $K$-theory of quantum automorphism groups of finite dimensional $C^{*}$-algebras in the sense of Wang. The results show in particular that the $C^{*}$-algebras of functions on the quantum permutation groups $S_{n}^{+}$are pairwise non-isomorphic for different values of $n$.

Along the way we discuss some general facts regarding torsion in discrete quantum groups. In fact, the duals of quantum automorphism groups are the most basic examples of discrete quantum groups exhibiting genuine quantum torsion phenomena.
\end{abstract}

\section{INTRODUCTION}

Quantum automorphism groups were introduced by Wang [29 in his study of noncommutative symmetries of finite dimensional $C^{*}$-algebras. These quantum groups are quite different from $q$-deformations of compact Lie groups, and interestingly, they appear naturally in a variety of contexts, including combinatorics and free probability, see for instance [7, 8]. The $C^{*}$-algebraic properties of quantum automorphism groups were studied by Brannan [11, revealing various similarities with free group $C^{*}$-algebras.

An interesting subclass of quantum automorphism groups is provided by quantum permutation groups. Following [9], we will write $S_{n}^{+}$for the quantum permutation group on $n$ letters. According to the definition of Wang, the quantum group $S_{n}^{+}$ is the universal compact quantum group acting on the abelian $C^{*}$-algebra $\mathbb{C}^{n}$. If one replaces $\mathbb{C}^{n}$ by a general finite dimensional $C^{*}$-algebra, one has to add the data of a state and restrict to state-preserving actions in the definition of quantum automorphism groups. Indeed, the choice of state is important in various respects. This is illustrated, for instance, by the work of De Rijdt and Vander Vennet on monoidal equivalences among quantum automorphism groups [13].

The aim of the present paper is to compute the $K$-theory of quantum automorphism groups. Our general strategy follows the ideas in 27, which in turn build on methods from the Baum-Connes conjecture, formulated in the language of category theory following Meyer and Nest [16]. In fact, the main result of [27] implies rather easily that the appropriately defined assembly map for duals of quantum automorphism groups is an isomorphism. The main additional ingredient, discussed further below, is the construction of suitable resolutions, entering the left hand side of the assembly map in the framework of [16].

The reason why this is more tricky than in 27] is that quantum automorphism groups have torsion. At first sight, the presence of torsion may appear surprising because these quantum groups behave like free groups in many respects. Indeed, the way in which torsion enters the picture is different from what happens for classical discrete groups. Therefore quantum automorphism groups provide an interesting

2000 Mathematics Subject Classification. 19D55, 81R50.

This work was supported by the Engineering and Physical Sciences Research Council Grant $\mathrm{EP} / \mathrm{L} 013916 / 1$. 
class of examples also from a conceptual point of view. Indeed, a better understanding of quantum torsion seems crucial in order to go beyond the class of quantum groups studied in the spirit of the Baum-Connes conjecture so far [17], 27], [26]. We have therefore included some basic considerations on torsion in discrete quantum groups in this paper.

From our computations discussed below one can actually see rather directly the effect of torsion on the level of $K$-theory. In particular, the $K$-groups of monoidally equivalent quantum automorphism groups can differ quite significantly due to minor differences in their torsion structure. Our results also have some direct operator algebraic consequences, most notably, they imply that the reduced $C^{*}$-algebras of functions on quantum permutation groups can be distinguished by $K$-theory.

Let us now explain how the paper is organised. In section 2 we collect some definitions and facts from the theory of compact quantum groups and fix our notation. Section 3 contains more specific preliminaries on quantum automorphism groups and their actions. In section 4 we collect some basic definitions and facts regarding torsion in discrete quantum groups. In the quantum case, this is studied most efficiently in terms of ergodic actions of the dual compact quantum groups, and our setup generalises naturally previous considerations by Meyer and Nest [17, [15]. Finally, section [5] contains our main results.

Let us conclude with some remarks on notation. We write $\mathbb{L}(\mathcal{E})$ for the algebra of adjointable operators on a Hilbert module $\mathcal{E}$. Moreover $\mathbb{K}(\mathcal{E})$ denotes the algebra of compact operators. The closed linear span of a subset $X$ of a Banach space is denoted by $[X]$. Depending on the context, the symbol $\otimes$ denotes either the tensor product of Hilbert spaces, the spatial tensor product of $C^{*}$-algebras, or the exterior tensor product of Hilbert modules.

\section{Compact quantum groups}

In this preliminary section we collect some definitions from the theory of compact quantum groups and fix our notation. We will mainly follow the conventions in [27] as far as general quantum group theory is concerned.

Let us start with the following definition.

Definition 2.1. A compact quantum group $G$ is given by a unital Hopf $C^{*}$-algebra $C(G)$, that is, a unital $C^{*}$-algebra $C(G)$ together with a unital *-homomorphism $\Delta: C(G) \rightarrow C(G) \otimes C(G)$, called comultiplication, such that

$$
(\Delta \otimes \mathrm{id}) \Delta=(\mathrm{id} \otimes \Delta) \Delta
$$

and

$$
[(C(G) \otimes 1) \Delta(C(G))]=C(G) \otimes C(G)=[(1 \otimes C(G)) \Delta(C(G))] .
$$

For every compact quantum group there exists a Haar state, namely a state $\phi$ : $C(G) \rightarrow \mathbb{C}$ satisfying the invariance conditions $(\mathrm{id} \otimes \phi) \Delta(f)=\phi(f) 1=(\phi \otimes \mathrm{id}) \Delta(f)$ for all $f \in C(G)$. The image of $C(G)$ in the GNS-representation of $\phi$ is denoted $C^{\mathrm{r}}(G)$, and called the reduced $C^{*}$-algebra of functions on $G$. We will write $L^{2}(G)$ for the GNS-Hilbert space of $\phi$, and notice that the GNS-representation of $C^{\mathrm{r}}(G)$ on $L^{2}(G)$ is faithful.

A unitary representation of $G$ on a Hilbert space $\mathcal{H}$ is a unitary element $U \in$ $M\left(C^{\mathrm{r}}(G) \otimes \mathbb{K}(\mathcal{H})\right)=\mathbb{L}\left(C^{\mathrm{r}}(G) \otimes \mathcal{H}\right)$ such that $(\Delta \otimes \mathrm{id})(U)=U_{13} U_{23}$. In analogy with the classical theory for compact groups, every unitary representation of a compact quantum group $G$ is completely reducible, and irreducible representations are finite dimensional. We write $\operatorname{Irr}(G)$ for the set of equivalence classes of irreducible unitary representations of $G$. The linear span of matrix coefficients of all unitary representations of $G$ forms a dense Hopf $*$-algebra $\mathcal{O}(G)$ of $C^{\mathrm{r}}(G)$.

The full $C^{*}$-algebra $C^{\mathrm{f}}(G)$ of functions on $G$ is the universal $C^{*}$-completion of 
$\mathcal{O}(G)$. It admits a comultiplication as well, satisfying the density conditions in definition 2.1. The quantum group $G$ can be equivalently described in terms of $C^{\mathrm{f}}(G)$ or $C^{\mathrm{r}}(G)$, or in fact, using $\mathcal{O}(G)$. One says that $G$ is coamenable if the canonical quotient map $C^{\mathrm{f}}(G) \rightarrow C^{\mathrm{r}}(G)$ is an isomorphism. In this case we will simply write again $C(G)$ for this $C^{*}$-algebra. By slight abuse of notation, we will also write $C(G)$ if a statement holds for both $C^{\mathrm{f}}(G)$ and $C^{\mathrm{r}}(G)$.

The regular representation of $G$ is the representation of $G$ on $L^{2}(G)$ corresponding to the multiplicative unitary $W \in M\left(C^{\mathrm{r}}(G) \otimes \mathbb{K}\left(L^{2}(G)\right)\right)$ determined by

$$
W^{*}(\Lambda(f) \otimes \Lambda(g))=(\Lambda \otimes \Lambda)(\Delta(g)(f \otimes 1)),
$$

where $\Lambda(f) \in L^{2}(G)$ is the image of $f \in C^{\mathrm{f}}(G)$ under the GNS-map. The comultiplication of $C^{\mathrm{r}}(G)$ can be recovered from $W$ by the formula

$$
\Delta(f)=W^{*}(1 \otimes f) W .
$$

One defines the algebra of functions $C_{0}(\hat{G})$ on the dual discrete quantum group $\hat{G}$ by

$$
C_{0}(\hat{G})=\left[\left(\mathbb{L}\left(L^{2}(G)\right)_{*} \otimes \mathrm{id}\right)(W)\right],
$$

together with the comultiplication

$$
\hat{\Delta}(x)=\hat{W}^{*}(1 \otimes x) \hat{W}
$$

for $x \in C_{0}(\hat{G})$, where $\hat{W}=\Sigma W^{*} \Sigma$. We remark that there is no need to distinguish between $C_{0}^{\mathrm{f}}(\hat{G})$ and $C_{0}^{\mathrm{r}}(\hat{G})$ in the discrete case.

Since we are following the conventions of Kustermans and Vaes 14, there is a flip map built into the above definition of $\hat{\Delta}$, so that the comultiplication of $C_{0}(\hat{G})$ corresponds to the opposite multiplication of $C^{\mathrm{r}}(G)$. This is a natural choice in various contexts, but it is slightly inconvenient when it comes to Takesaki-Takai duality. We will write $\check{G}$ for $C_{0}(\hat{G})^{\text {cop }}$, that is, for the Hopf $C^{*}$-algebra $C_{0}(\hat{G})$ equipped with the opposite comultiplication $\hat{\Delta}^{\text {cop }}=\sigma \hat{\Delta}$, where $\sigma$ denotes the flip map. By slight abuse of terminology, we shall refer to both $\check{G}$ and $\hat{G}$ as the dual quantum group of $G$, but in the sequel we will always work with $\check{G}$ instead of $\hat{G}$. According to Pontrjagin duality, the double dual of $G$ in either of the two conventions is canonically isomorphic to $G$.

An action of a compact quantum group $G$ on a $C^{*}$-algebra $A$ is a coaction of $C(G)$ on $A$, that is, an injective nondegenerate $*$-homomorphism $\alpha: A \rightarrow M(C(G) \otimes A)$ such that $(\Delta \otimes \mathrm{id}) \alpha=(\mathrm{id} \otimes \alpha) \alpha$ and $[(C(G) \otimes 1) \alpha(A)]=C(G) \otimes A$. In a similar way one defines actions of discrete quantum groups, or in fact arbitrary locally compact quantum groups. We will call a $C^{*}$-algebra equipped with a coaction of $C^{\mathrm{r}}(G)$ a $G$ - $C^{*}$-algebra. Moreover we write $G$-Alg for the category of all $G$ - $C^{*}$-algebras and equivariant $*$-homomorphisms.

The reduced crossed product $G \ltimes_{\mathrm{r}} A$ of a $G$ - $C^{*}$-algebra $A$ is the $C^{*}$-algebra

$$
G \ltimes_{\mathrm{r}} A=\left[\left(C_{0}(\check{G}) \otimes 1\right) \alpha(A)\right]
$$

The crossed product is equipped with a canonical dual action of $\check{G}$, which turns it into a $\check{G}$ - $C^{*}$-algebra. Moreover, one has the following analogue of the TakesakiTakai duality theorem [1].

Theorem 2.2. Let $G$ be a regular locally compact quantum group and let $A$ be a $G-C^{*}$-algebra. Then there is a natural isomorphism

$$
\left.\check{G} \ltimes_{\mathrm{r}} G \ltimes_{\mathrm{r}} A \cong \mathbb{K}\left(L^{2}(G)\right)\right) \otimes A
$$

of $G-C^{*}$-algebras. 
We will use Takesaki-Takai duality only for discrete and compact quantum groups, and in this setting regularity is automatic. At some points we will also use the full crossed product $G \ltimes_{\mathrm{f}} A$ of a $G$ - $C^{*}$-algebra $A$, and we refer to [20] for a review of its definition in terms of its universal property for covariant representations.

\section{Quantum Automorphism groups}

In this section we review some basic definitions and results on quantum automorphism groups of finite dimensional $C^{*}$-algebras and fix our notation. We refer to [29, 3], 4] for more background on quantum automorphism groups.

Let us start with the definition of the quantum automorphism group of a finite dimensional $C^{*}$-algebra $A$, compare [29] [3]. If $\mu: A \otimes A \rightarrow A$ denotes the multiplication map then a faithful state $\omega$ on $A$ is called a $\delta$-form for $\delta>0$ if $\mu \mu^{*}=\delta^{2}$ id with respect to the Hilbert space structures on $A$ and $A \otimes A$ implemented by the GNS-constructions for $\omega$ and $\omega \otimes \omega$, respectively.

Definition 3.1. Let $A$ be a finite dimensional $C^{*}$-algebra and let $\omega$ be a $\delta$-form on $A$ for some $\delta>0$. The quantum automorphism group $\operatorname{Qut}(A)=\operatorname{Qut}(A, \omega)$ is the universal compact quantum group acting on $A$ such that $\omega$ is preserved.

That is, if $G$ is any compact quantum group together with a coaction $\delta: A \rightarrow$ $C^{\mathrm{f}}(G) \otimes A$ then there exists a unique morphism of quantum groups $\iota: G \rightarrow \operatorname{Qut}(A)$ such that the diagram

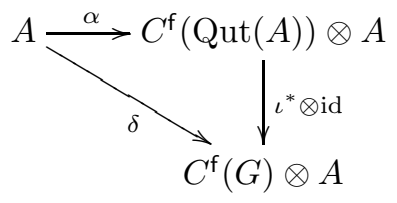

is commutative and

$$
(\mathrm{id} \otimes \omega) \alpha(a)=\omega(a) 1
$$

for all $a \in A$. Here $\iota^{*}: C^{\mathrm{f}}(\operatorname{Qut}(A)) \rightarrow C^{\mathrm{f}}(G)$ denotes the homomorphism of Hopf $C^{*}$-algebras corresponding to the morphism $\iota$.

A basic result of the theory is that the compact quantum group $\operatorname{Qut}(A, \omega)$ indeed exists [29], see also [19]. As indicated in definition 3.1, we will typically omit the state $\omega$ from our notation and write $\operatorname{Qut}(A)$ instead of $\operatorname{Qut}(A, \omega)$, although $\omega$ is an important part of the data. The notation for quantum automorphism groups used in 29 is $A_{\text {aut }}(B)=C^{\mathrm{f}}(\operatorname{Qut}(B))$.

We remark that the matrix coefficients of an action of a compact quantum group $G$ on a finite dimensional $C^{*}$-algebra $A$ are contained in the Hopf $*$-algebra $\mathcal{O}(G)$. In particular, any coaction $\alpha: A \rightarrow C^{\mathrm{f}}(G) \otimes A$, where $G$ is a compact quantum group, comes from a Hopf algebraic coaction $\alpha: A \rightarrow \mathcal{O}(G) \otimes A$. Using this fact, one can study quantum automorphism groups from a more algebraic perspective, see for instance [19. The universal $C^{*}$-algebras of quantum automorphism groups can be defined explicitly in terms of generators and relations [29].

Let us take a closer look at the special case of quantum permutation groups. By definition, the quantum permutation group $S_{n}^{+}$is the quantum automorphism group of $A=\mathbb{C}^{n}$ with the trace corresponding to the uniform probability measure on $n$ points, which is a $\delta$-form for $\delta=\sqrt{n}$. In order to describe $C^{\mathrm{f}}\left(S_{n}^{+}\right)$let us recall some terminology. If $B$ is any unital $*$-algebra then a matrix $u=\left(u_{i j}\right) \in M_{n}(B)$ is called a magic unitary if all entries $u_{i j}$ are projections, and on each row and column of $u$ these projections are mutually orthogonal and sum up to 1. Explicitly, this means

$$
u_{i j}^{*}=u_{i j}=u_{i j}^{2}
$$


for all $1 \leq i, j \leq n$ and

$$
\sum_{i=1}^{n} u_{i k}=1, \quad \sum_{i=1}^{n} u_{k i}=1
$$

for all $k$. These relations imply in particular that the matrix $u$ and its transpose $u^{t}$ are both unitary.

Proposition 3.2. The full $C^{*}$-algebra of functions on the quantum permutation group $S_{n}^{+}=\operatorname{Qut}\left(\mathbb{C}^{n}\right)$ is the universal unital $C^{*}$-algebra $C^{\mathrm{f}}\left(S_{n}^{+}\right)$with generators $u_{i j}$ for $1 \leq i, j \leq n$ such that $u=\left(u_{i j}\right) \in M_{n}\left(C^{\mathrm{f}}\left(S_{n}^{+}\right)\right)$is a magic unitary matrix.

The comultiplication $\Delta: C^{\mathrm{f}}\left(S_{n}^{+}\right) \rightarrow C^{\mathrm{f}}\left(S_{n}^{+}\right) \otimes C^{\mathrm{f}}\left(S_{n}^{+}\right)$is defined by the formula

$$
\Delta\left(u_{i j}\right)=\sum_{k=1}^{n} u_{i k} \otimes u_{k j}
$$

on generators. The defining coaction $\alpha: \mathbb{C}^{n} \rightarrow C^{\mathrm{f}}\left(S_{n}^{+}\right) \otimes \mathbb{C}^{n}$ is given by

$$
\alpha\left(e_{i}\right)=\sum_{j=1}^{n} u_{i j} \otimes e_{j},
$$

where $e_{1}, \ldots, e_{n}$ are the minimal projections in $\mathbb{C}^{n}$.

From the fact that $S_{n}^{+}$is the universal compact quantum group acting on $A=\mathbb{C}^{n}$ we obtain a morphism of quantum groups $S_{n} \rightarrow S_{n}^{+}$, that is, a unital $*$-homomorphism $C^{\mathrm{f}}\left(S_{n}^{+}\right) \rightarrow C\left(S_{n}\right)$ compatible with comultiplications. Here $S_{n}$ is the symmetric group acting by permutations on $A$. In fact, every character of $C^{\mathrm{f}}\left(S_{n}^{+}\right)$is induced from a character of $C\left(S_{n}\right)$, and $C\left(S_{n}\right)$ is the abelianisation of $C^{\mathrm{f}}\left(S_{n}^{+}\right)$.

The structure of quantum permutation groups $S_{n}^{+}$is well-understood for small values of $n$, for the following fact compare [5].

Lemma 3.3. For $n=1,2,3$ the canonical morphism of quantum groups $S_{n} \rightarrow S_{n}^{+}$ is an isomorphism.

Clearly, this means in particular that $S_{n}^{+}$is coamenable for these values of $n$. For $n=4$ the natural morphism $S_{n} \rightarrow S_{n}^{+}$is no longer an isomorphism. In fact, the $C^{*}$-algebra $C^{\mathrm{f}}\left(S_{4}^{+}\right)$is infinite dimensional. The following result due to Banica and Bichon [6] describes the structure of $S_{4}^{+}$.

Theorem 3.4. There is an isomorphism of quantum groups $S_{4}^{+} \cong S_{-1}(3)$, where the latter is obtained from $S_{-1}(3)$ by making the fundamental matrix orthogonal. The quantum group $S_{-1}(3)$ is a 2-cocycle twist of the classical group $S O(3)$.

We note that since the classical group $S O(3)$ is a coamenable compact quantum group, the same holds for its cocycle twist $S_{4}^{+}$, see [2]. For $n \geq 5$ the quantum groups $S_{n}^{+}$are no longer coamenable, that is, the reduced $C^{*}$-algebras $C^{\mathrm{r}}\left(S_{n}^{+}\right)$fail to be nuclear.

Still, the $C^{*}$-algebras $C^{\mathrm{r}}\left(S_{n}^{+}\right)$are exact for all $n$. This can be shown using the monoidal equivalences among quantum automorphism groups [13] to be discussed below, by invoking a general observation of Vaes and Vergnioux [23, namely that exactness of the reduced $C^{*}$-algebras of functions on compact quantum groups is preserved under monoidal equivalences, compare [11.

According to lemma 3.3 all quantum automorphisms for $C^{*}$-algebras of dimension at most 3 come from classical automorphisms. In the sequel we will therefore restrict attention to finite dimensional $C^{*}$-algebras of dimension at least 4 .

In the case of dimension 4 , the only $C^{*}$-algebra to consider apart from $\mathbb{C}^{4}$ is $M_{2}(\mathbb{C})$. The quantum automorphism group of $A=\mathbb{C}^{4}$ is the quantum permutation group $S_{4}^{+}$already mentioned above, and for $M_{2}(\mathbb{C})$, the quantum automorphism groups are determined by the following result of Soltan $[22]$. 
Theorem 3.5. Let $\omega$ be a faithful state on $M_{2}(\mathbb{C})$. The quantum automorphism group $\operatorname{Qut}\left(M_{2}(\mathbb{C}), \omega\right)$ is isomorphic to $S O_{q}(3)$ for a unique $q \in(0,1]$. Here $S O_{q}(3)$ is the quantum $S O(3)$-group of Podlè̀.

We emphasise that the quantum group $S O_{q}(3)$ of Podles̀ [21] is different from the quantum group $S_{-1}(3)$ appearing in theorem 3.4 .

Let us now return to general quantum automorphism groups. The main tool at our disposal are the monoidal equivalences exhibited by De Rijdt and Vander Vennet as follows 13 .

Theorem 3.6. Let $A_{j}$ be finite dimensional $C^{*}$-algebras of dimension at least 4 , equipped with $\delta_{j}$-forms $\omega_{j}$ for $j=1,2$, respectively. Then $\operatorname{Qut}\left(A_{1}\right)$ is monoidally equivalent to $\operatorname{Qut}\left(A_{2}\right)$ iff $\delta_{1}=\delta_{2}$.

This result will play a crucial role in our analysis, it implies in particular that any quantum automorphism group is monoidally equivalent to $S O_{q}(3)$ for some $q \in(0,1]$. Theorem 3.6 shows also that the quantum permutation groups $S_{n}^{+}=$ Qut $\left(\mathbb{C}^{n}\right)$ are pairwise monoidally inequivalent for $n \geq 4$.

\section{TORSION IN DISCRETE QUANTUM GROUPS}

In this section we discuss some definitions and facts related to torsion in discrete quantum groups. This will be useful in our analysis of the equivariant Kasparov theory of quantum automorphism groups. The study of torsion in duals of compact groups has already been carried out in [17, and our definitions are by and large motivated from this.

Firstly, we have to explain what we mean by torsion. Let $\breve{G}$ be the dual of a discrete quantum group $G$, and assume that $U \in C^{\mathrm{r}}(\check{G}) \otimes \mathbb{K}(V)$ is a unitary representation of $\breve{G}$ on the finite dimensional Hilbert space $V$. Then we obtain a coaction $\operatorname{ad}_{U}$ : $\mathbb{K}(V) \rightarrow C^{\mathrm{r}}(\check{G}) \otimes \mathbb{K}(V)$ by the formula

$$
\operatorname{ad}_{U}(T)=U^{*}(\operatorname{id} \otimes T) U,
$$

which turns $\mathbb{K}(\mathcal{H})$ into a $\check{G}$ - $C^{*}$-algebra. Coactions of this form are precisely the coactions on simple matrix algebras which are $\check{G}$-equivariantly Morita equivalent to the trivial coaction on $\mathbb{C}$.

The idea is, roughly, that torsion in $G$ is encoded in coactions on finite dimensional $C^{*}$-algebras which are not of the above form. In order to make this precise, recall that a unital $\check{G}$ - $C^{*}$-algebra $B$ with coaction $\beta: B \rightarrow C(\check{G}) \otimes B$ is called ergodic iff its fixed point subalgebra

$$
B^{\beta}=\{b \in B \mid \beta(b)=1 \otimes b\}
$$

is equal to $\mathbb{C}$.

The following definition is motivated by the considerations regarding torsion-free quantum groups in [15].

Definition 4.1. Let $G$ be a discrete quantum group. A finite dimensional ergodic $\check{G}-C^{*}$-algebra $B$ is called a torsion coaction of $G$. Such a coaction is called nontrivial if $B$ is not $\breve{G}$-equivariantly Morita equivalent to the trivial $\check{G}-C^{*}$-algebra $\mathbb{C}$.

The quantum group $G$ is called torsion-free if $G$ does not admit any nontrivial torsion coactions.

It is straightforward to check that a quantum group $G$ is torsion-free iff for every finite dimensional $\breve{G}$ - $C^{*}$-algebra $B$ there are finite dimensional unitary corepresentations $U_{j} \in C^{\mathrm{r}}(\check{G}) \otimes \mathbb{K}\left(V_{j}\right)$ such that

$$
B \cong \mathbb{K}\left(V_{1}\right) \oplus \cdots \oplus \mathbb{K}\left(V_{l}\right)
$$


as $\check{G}$ - $C^{*}$-algebras, where each matrix block $\mathbb{K}\left(V_{j}\right)$ is equipped with the adjoint coaction $\operatorname{ad}_{U_{j}}$ as explained above. That is, the notion of torsion-freeness in definition 4.1 is compatible with the terminology used in [15], 27.

Assume that $G$ is a discrete quantum group, and let $Q$ be a Galois object for $C^{*}(H)^{\text {cop }}=C(\check{H})$ where $H \subset G$ is a finite quantum subgroup. That is, $Q$ is a $C^{*}$ algebra equipped with an ergodic coaction of $C(\check{H})$ of full quantum multiplicity, compare for instance [10, 12. Then $Q$ is a torsion coaction of $G$ since $Q$ is clearly finite dimensional, and the ergodic action of $\check{H}$ on $Q$ is naturally an ergodic action of $\check{G}$ as well.

The following proposition shows that this class of coactions exhausts all torsion coactions in the case of classical discrete groups.

Proposition 4.2. Let $G$ be a discrete group. Then every torsion coaction of $G$ is $\check{G}$-equivariantly Morita equivalent to a $\check{G}$ - $C^{*}$-algebra of the form $C_{\omega}^{*}(H)$ for some finite subgroup $H \subset G$ and a normalised cocycle $\omega \in Z^{2}(H, U(1))$.

Proof. Let $\beta: B \rightarrow C_{\mathrm{r}}^{*}(G) \otimes B$ be a torsion coaction and consider the corresponding spectral decomposition

$$
B=\bigoplus_{s \in G} B_{s}
$$

of $B$, where $B_{s}=\{b \in B \mid \beta(b)=s \otimes b\}$. Ergodicity means that the component of the identity element $e \in G$ is $B_{e}=\mathbb{C}$. Observe next that if $b \in B_{s}$ then $b^{*} \in B_{s}^{-1}$ and $b^{*} b \in B_{e}$, so that $b^{*} b=\lambda>0$ is invertible. It follows that all spectral subspaces are one-dimensional and spanned by invertible elements.

For every $s \in G$ such that $B_{s}$ is nonzero we may choose a unitary $\delta_{s} \in B_{s}$, and without loss of generality we may pick $\delta_{e}=1$. Clearly, the elements $s \in G$ such that $B_{s} \neq 0$ form a finite subgroup $H$ of $G$. Moreover we have $\delta_{s} \delta_{t}=\omega(s, t) \delta_{s t}$ for $\omega(s, t) \in U(1)$. It is straightforward to check that this yields a normalised 2-cocycle $\omega \in Z^{2}(H, U(1))$, and we conclude that $B$ is isomorphic to $C_{\omega}^{*}(H)$, the twisted group $C^{*}$-algebra of $H$.

As a corollary of proposition 4.2 we see in particular that the notion of torsionfreeness introduced in definition 4.1 agrees with the usual terminology for discrete groups. That is, a discrete group $G$ is torsion-free in the sense of definition 4.1 iff it has no nontrivial finite subgroups.

In a sense, the torsion coactions obtained from Galois objects for finite quantum subgroups are the most obvious examples of torsion coactions. If one goes beyond classical discrete groups then more exotic torsion coactions can appear.

In particular, as shown in [17, this already happens for duals of compact groups. If $G$ is a compact group then a torsion coaction of the dual discrete quantum group $\check{G}$ is nothing but an ergodic action of $G$ on a finite dimensional $C^{*}$-algebra $B$. Such actions factorise over a Lie group quotient $K$ of $G$ because the group $\operatorname{Aut}(B)$ of *-automorphisms of $B$ is a compact Lie group. Moreover, ergodicity implies that $B$ must consist of matrix blocks of the same size. That is, we have $B \cong M_{k}(\mathbb{C})^{\oplus n}$ for some $k, n \in \mathbb{N}$. The subgroup $L \subset K$ preserving a fixed matrix block of $B$ contains the connected component $K_{(0)}$ of $K$, and $B$ is isomorphic to the induced $C^{*}$-algebra $\operatorname{ind}_{L}^{K}\left(M_{k}(\mathbb{C})\right)$ corresponding to the action of $L$ on $M_{k}(\mathbb{C})$. In other words, we see in particular that all proper homogeneous $\breve{G}-C^{*}$-algebras considered in [17] arise from torsion coactions of $\breve{G}$.

It is sometimes useful to distinguish between two basic types of torsion coactions. Let us say that a projective torsion coaction of a discrete quantum group $G$ is a torsion coaction whose underlying $C^{*}$-algebra is simple, and which is not equivariantly Morita equivalent to $\mathbb{C}$. Moreover, let us refer to all torsion coactions on non-simple $C^{*}$-algebras as permutation torsion coactions. Clearly, the quantum 
group $G$ is torsion-free if it has neither projective torsion nor permutation torsion. The terminology is motivated from the fact that one sourse of permutation torsion for $G$ comes from finite quantum subgroups and their regular coactions. Similarly, projective torsion arises from projective representations of $\check{G}$.

Roughly speaking, the following lemma shows that permutation torsion for $G$ is related to the connectedness of $\breve{G}$.

Lemma 4.3. The dual of a compact group $G$ has no permutation torsion iff $G$ is connected.

Proof. If $G$ is connected, then every action of $G$ on

$$
B=M_{k_{1}}(\mathbb{C}) \oplus \cdots \oplus M_{k_{n}}(\mathbb{C})
$$

must preserve the individual matrix blocks, and ergodicity implies that $n=1$. Conversely, assume that $G$ is not connected. Then the quotient $G / G_{(0)}$ of $G$ by its connected component is a nontrivial profinite group. If $F$ is a nontrivial finite quotient of $G / G_{(0)}$, then the permutation action of $F$ on the commutative $C^{*}$-algebra $C(F)$ induces an ergodic action of $G$ via the quotient maps $G \rightarrow G / G_{(0)} \rightarrow F$. Hence $G$ has permutation torsion in this case.

Assume that $G$ and $H$ are discrete quantum groups such that $\check{G}$ and $\check{H}$ are monoidally equivalent. Then the general correspondence between actions of $\check{G}$ and $\check{H}$ shows that torsion coactions of $G$ and $H$ are in a bijective correspondence [13, [27]. For duals of quantum automorphism groups it is therefore quite easy to determine all torsion coactions up to equivariant Morita equivalence.

Lemma 4.4. Let $\operatorname{Qut}(A, \omega)$ be the quantum automorphism group of a $C^{*}$-algebra $A$ of dimension at least 4 with respect to the $\delta$-form $\omega$. Then the trivial coaction on $\mathbb{C}$ and the defining coaction on $A$ are the only torsion coactions of the dual of $\operatorname{Qut}(A, \omega)$ up to equivariant Morita equivalence.

Proof. According to the results in [13, the quantum group $\operatorname{Qut}(A, \omega)$ is monoidally equivalent to $H=S O_{q}(3)$ for some $q \in(0,1]$. If we write $G=S U_{q}(2)$, then we have $C(H) \subset C(G)$, and if $B$ is a finite dimensional ergodic $H$ - $C^{*}$-algebra, it is also naturally an ergodic $G$ - $C^{*}$-algebra. According to [27, the quantum group $\check{G}$ is torsion-free. It follows that $B \cong \mathbb{K}(V(n))$ for some $n \in \frac{1}{2} \mathbb{N}_{0} \cong \operatorname{Irr}\left(S U_{q}(2)\right)$. If $n$ is an integer then $B$ is $H$-equivariantly Morita equivalent to $\mathbb{C}$. In this case the corresponding ergodic action of $\operatorname{Qut}(A, \omega)$ is Morita equivalent to the trivial action on $\mathbb{C}$. Otherwise $B$ is $H$-equivariantly Morita equivalent to $\mathbb{K}(V(1 / 2))$. The $H$ $C^{*}$-algebra $\mathbb{K}(V(1 / 2))$ corresponds to $A$ under the monoidal equivalence, because up to isomorphism it is the only ergodic $H$ - $C^{*}$-algebra with the correct spectral decomposition.

In particular, lemma 4.4 shows that projective torsion may be turned into permutation torsion under monoidal equivalence, and vice versa.

For the applications to discrete quantum groups we have to study the crossed products of torsion coactions, and it will be convenient to use the following terminology, compare [17.

Definition 4.5. Let $G$ be a discrete quantum group. A $G$-C $C^{*}$-algebra is called proper almost homogeneous if it is G-equivariantly Morita equivalent to the crossed product of some torsion coaction of $G$.

Notice that we do not need to distinguish between reduced or maximal crossed products here since the dual of $G$ is compact.

A guiding example for proper almost homogeneous algebras arises from the torsion coaction $B=C(\check{H})$ for some finite quantum subgroup $H \subset G$. In this case the proper almost homogeneous algebra $G \ltimes B$ is $G$-equivariantly Morita equivalent to 
$C_{0}(G / H)$, which should be viewed as the prototypical example of a proper homogeneous action of $G$.

According to proposition 4.2 proper almost homogeneous actions of classical discrete groups are indeed essentially determined by homogeneous spaces $G / H$ where $H$ is finite. Notice that in the presence of a nontrivial cocycle $\omega \in Z^{2}(H, U(1))$, the crossed product $P=\breve{G} \ltimes C_{\omega}^{*}(H)$ is a proper $G$ - $C^{*}$-algebra over $C_{0}(G / H)$ in the sense of Kasparov.

Assume that $G$ be a locally compact quantum group and let $A$ be a $G$ - $C^{*}$-algebra. Let us say that $G$ acts amenably on $A$ if the canonical quotient map $G \ltimes_{\mathrm{f}} A \rightarrow G \ltimes_{\mathrm{r}} A$ is an isomorphism. In particular, according to this terminology, $G$ is amenable in the sense that $C_{\mathrm{f}}^{*}(G) \cong C_{\mathrm{r}}^{*}(G)$ iff $G$ acts amenably on $\mathbb{C}$.

Lemma 4.6. Let $G$ be a discrete quantum group. If $P$ is a proper almost homogeneous $G$ - $C^{*}$-algebra then $G$ acts amenably on $P$.

Proof. Without loss of generality we may assume that $P=\check{G} \ltimes B$ for a finite dimensional $C^{*}$-algebra $B$ with an ergodic action of $\check{G}$. It is enough to observe that the algebraic crossed product $G \ltimes_{\text {alg }} \check{G} \ltimes_{\text {alg }} B$, taken in the framework of algebraic quantum groups [24, is dense inside both $G \ltimes_{\mathrm{f}} P$ and $G \ltimes_{\mathrm{r}} P$. Indeed, the algebraic crossed product is isomorphic to the algebraic tensor product of $B$ with an algebra of possibly infinite matrices, and the $C^{*}$-norm on such an algebra is uniquely determined. Therefore the quotient map $G \ltimes_{\mathrm{f}} P \rightarrow G \ltimes_{\mathrm{r}} P$ is an isomorphism.

\section{The $K$-THEORY OF QUANTUM AUTOMORPHISM GROUPS}

In this section we compute the $K$-theory of quantum automorphism groups. The basic strategy is the same as for free quantum groups [27, [26], namely, in a first step it will be shown that quantum automorphism groups satisfy a strong form of the Baum-Connes conjecture. The second step consists of the actual computation, essentially this amounts to computing the left hand side of the assembly map.

Let $G=\operatorname{Qut}(A, \omega)$ be the quantum automorphism group of a finite dimensional $C^{*}$-algebra $A$ with respect to a $\delta$-form $\omega$. In the sequel we will always assume that the dimension of $A$ is at least 4 . We will also fix $q \in(0,1]$ such that $H=S O_{q}(3)$ is monoidally equivalent to $G$, see [13].

Firstly, we have to analyse the structure of the equivariant $K K$-theory of $G$. For this we need the language of triangulated categories, compare [16, [20, 27. More precisely, we consider the category $K K^{G}$ with objects all separable $G$ - $C^{*}$-algebras, and the morphism set between objects $B$ and $C$ is given by the equivariant Kasparov group $K K^{G}(B, C)$. Composition of morphisms is given by the Kasparov product. The category $K K^{G}$ is triangulated with translation automorphism $\Sigma: K K^{G} \rightarrow$ $K K^{G}$ given by the suspension $\Sigma B=C_{0}(\mathbb{R}, B)$ of a $G$ - $C^{*}$-algebra $B$. The exact triangles are all diagrams isomorphic to diagrams of the form<smiles>[CH]B[CH][CH]</smiles>

where $C_{f}$ denotes the mapping cone of an equivariant $*$-homomorphism $f: B \rightarrow C$. For more information we refer to [16, [20, [27.

Let $\mathcal{T}_{G} \subset K K^{G}$ be the full subcategory consisting of all trivial $G$ - $C^{*}$-algebras, and all $G$ - $C^{*}$-algebras of the form $A \otimes C$, equipped with the the action coming from the defining action of $G$ on the first tensor factor. These actions should be thought of as crossed products of compactly induced actions for the dual discrete quantum group $\check{G}$. We write $\left\langle\mathcal{T}_{G}\right\rangle$ for the localising subcategory of $K K^{G}$ generated by $\mathcal{T}_{G}$. Accordingly, we let $\left\langle\mathcal{C I}_{\breve{G}}\right\rangle \subset K K^{\check{G}}$ be the full subcategory corresponding to $\left\langle\mathcal{T}_{G}\right\rangle$ under Baaj-Skandalis duality [1, that is, under the equivalence $K K^{G} \rightarrow K K^{\check{G}}$ of triangulated categories given by taking reduced crossed products. In the sequel we 
will however avoid working in $K K^{\check{G}}$ for most of the time, because the constructions are somewhat clearer in the compact picture.

Since $H=S O_{q}(3)$ is monoidally equivalent to $G$, we have an equivalence of triangulated categories between $K K^{H}$ and $K K^{G}$, see [27]. Notice that the category $\mathcal{T}_{G}$ corresponds to $\mathcal{T}_{H}$ under this equivalence. Therefore, it largely suffices to study the structure of $K K^{H}$.

Recall that the quantum group $H=S O_{q}(3)$ is closely related to $K=S U_{q}(2)$ since $C\left(S O_{q}(3)\right) \subset C\left(S U_{q}(2)\right)$. We will identify the set $\operatorname{Irr}\left(S U_{q}(2)\right)$ of equivalence classes of irreducible representations of $S U_{q}(2)$ with $\frac{1}{2} \mathbb{N}_{0}$, and refer to the irreducible representation $V(n)$ of $S U_{q}(2)$ corresponding to $n \in \frac{1}{2} \mathbb{N}_{0}$ as the representation of spin $n$. In the Peter-Weyl picture, the algebra $C\left(S O_{q}(3)\right)$ is the norm closure of the space of matrix elements of all integral spin representations. Accordingly, the set $\operatorname{Irr}\left(S O_{q}(3)\right)$ of irreducible representations of $S O_{q}(3)$ identifies with $\mathbb{N}_{0}$. We shall write $C_{\omega}^{*}\left(S O_{q}(3)\right)$ for the quotient of $C^{*}\left(S U_{q}(2)\right)$ corresponding to the remainig part of $\operatorname{Irr}\left(S U_{q}(2)\right)$. That is, $C_{\omega}^{*}\left(S O_{q}(3)\right)$ is the norm closure of the matrix algebras associated to representations of half-integral spin. By construction, we have a direct sum decomposition

$$
C^{*}\left(S U_{q}(2)\right)=C^{*}\left(S O_{q}(3)\right) \oplus C_{\omega}^{*}\left(S O_{q}(3)\right),
$$

and this decomposition is compatible with the canonical coactions of $C^{*}\left(S O_{q}(3)\right)$ induced from the comultiplication of $C^{*}\left(S U_{q}(2)\right)$.

Lemma 5.1. There is a $C^{*}\left(S O_{q}(3)\right)$-colinear Morita equivalence

$$
C_{\omega}^{*}\left(S O_{q}(3)\right) \sim S O_{q}(3) \ltimes \mathbb{K}(V(1 / 2))
$$

where $V(1 / 2)$ is the defining representation of $S U_{q}(2)$.

Proof. We simply have to cut down the $C^{*}\left(S U_{q}(2)\right)$-colinear Morita equivalence between $C^{*}\left(S U_{q}(2)\right)$ and $S U_{q}(2) \ltimes \mathbb{K}(V(1 / 2))$ implemented by the imprimitivity bimodule

$$
C^{*}\left(S U_{q}(2)\right) \ltimes V(1 / 2)=\left[\left(C^{*}\left(S U_{q}(2)\right) \otimes 1\right) \lambda(V(1 / 2))\right] \subset \mathbb{L}\left(L^{2}\left(S U_{q}(2)\right) \otimes V(1 / 2)\right) .
$$

More precisely, split the Hilbert space $L^{2}\left(S U_{q}(2)\right)=\mathcal{H}_{0} \oplus \mathcal{H}_{1}$ into the direct sum of $\mathcal{H}_{0}=L^{2}\left(S O_{q}(3)\right)$ and its orthogonal complement $\mathcal{H}_{1}$. Then we obtain a Hilbert $C_{\omega}^{*}\left(S O_{q}(3)\right)$-module

$$
\left[\left(C^{*}\left(S O_{q}(3)\right) \otimes 1\right) \lambda(V(1 / 2))\left(C_{\omega}^{*}\left(S O_{q}(3)\right) \otimes 1\right)\right] \subset \mathbb{L}\left(\mathcal{H}_{1}, \mathcal{H}_{0}\right) \otimes V(1 / 2),
$$

and it is straightforward to check that this module implements a $C^{*}\left(S O_{q}(3)\right)$ colinear Morita equivalence between $C_{\omega}^{*}\left(S O_{q}(3)\right)$ and $S O_{q}(3) \ltimes \mathbb{K}(V(1 / 2))$.

The advantage of $S O_{q}(3) \ltimes \mathbb{K}(V(1 / 2))$ over $C_{\omega}^{*}\left(S O_{q}(3)\right)$ is that it is easy to check what happens to the former under monoidal equivalences. Indeed, according to proposition 4.4, the $S O_{q}(3)-C^{*}$-algebra $\mathbb{K}(V(1 / 2))$ corresponds to the defining action of $G=\operatorname{Qut}(A)$ on $A$.

Recall that a discrete quantum group $F$ is called $K$-amenable if the canonical map $F \ltimes_{\mathrm{f}} B \rightarrow F \ltimes_{\mathrm{r}} B$ is an isomorphism in $K K$ for any $F-C^{*}$-algebra $B$, compare 25. The following theorem shows that duals of quantum automorphism groups satisfy the Baum-Connes conjecture.

Theorem 5.2. Let $A$ be a finite dimensional $C^{*}$-algebra of dimension $\operatorname{dim}(A) \geq 4$, and let $G$ be the quantum automorphism group of $A$ with respect to a $\delta$-form on $A$. Then we have $K K^{G}=\left\langle\mathcal{T}_{G}\right\rangle$. In particular, the dual of $G$ is $K$-amenable.

Proof. For the first claim it is enough to check the corresponding assertion for $H=S O_{q}(3)$. In this case it follows from the strong Baum-Connes property of $K=S U_{q}(2)$, see [27]. More precisely, let $B \in K K^{\check{H}}$ and consider the induction 
functor $K K^{\check{H}} \rightarrow K K^{\check{K}}$. According to 27, the algebra ind $\check{K}_{\breve{H}}^{\check{L}}(B)$ is contained in the localising subcategory of $K K^{\check{K}}$ generated by all algebras of the form $C_{0}(\check{K}) \otimes C$, where $C$ is any $C^{*}$-algebra, with the coaction given by comultiplication on the first tensor factor. Moreover, due to lemma [5.1, the $\check{H}$ - $C^{*}$-algebra $C_{0}(\check{K})$ decomposes as

$$
C_{0}(\check{K}) \cong\left(S O_{q}(3) \ltimes \mathbb{C}\right) \oplus\left(S O_{q}(3) \ltimes \mathbb{K}(V(1 / 2))\right)
$$

in $K K^{\check{H}}$. Therefore it suffices to observe that $B$ is a direct summand in $\operatorname{res}_{\breve{H}}^{\check{K}} \operatorname{ind}_{\breve{H}}^{\check{K}}(B)$ as an $\check{H}$-algebra. Using the concrete description of induced $C^{*}$-algebras given in 26, this in turn follows by considering the coaction of $B$, viewed as a $*-$ homomorphism $\beta: B \rightarrow \operatorname{ind}_{\check{H}}^{\check{K}}(B) \subset M\left(C_{0}(\hat{K}) \otimes B\right)$, and the map $\check{\epsilon} \otimes$ id : $\operatorname{ind}_{\check{H}}^{\check{K}}(B) \rightarrow B$ where $\check{\epsilon}$ denotes the counit of $C_{0}(\check{K})$.

The $K$-amenability of $\breve{G}$ follows now immediately using lemma 4.6, compare the analogous argument in 27 .

If $C$ is an $S O_{q}(3)-C^{*}$-algebra we write $\mathbb{K}(V(1 / 2)) \otimes C$ for the $S O_{q}(3)$-algebra obtained by considering the $S U_{q}(2)-C^{*}$-algebra $\mathbb{K}(V(1 / 2)) \otimes C$ and observing that the coaction on $\mathbb{K}(V(1 / 2)) \otimes C$ takes values in $C\left(S O_{q}(3)\right) \otimes \mathbb{K}(V(1 / 2)) \otimes C$.

Lemma 5.3. For any $S O_{q}(3)-C^{*}$-algebra $B$ we have an $S O_{q}(3)$-equivariant Morita equivalence

$$
\mathbb{K}(V(1 / 2)) \otimes \mathbb{K}(V(1 / 2)) \otimes B \sim_{M} B .
$$

Moreover, there exists a natural isomorphism

$$
K K^{S O_{q}(3)}(\mathbb{K}(V(1 / 2)) \otimes B, \mathbb{K}(V(1 / 2)) \otimes C) \cong K K^{S O_{q}(3)}(B, C)
$$

for all $S O_{q}(3)-C^{*}$-algebras $B, C$.

Proof. For the first claim observe that

$$
\mathbb{K}(V(1 / 2)) \otimes \mathbb{K}(V(1 / 2)) \otimes B \cong \mathbb{K}(V(1 / 2) \otimes V(1 / 2)) \otimes B
$$

is $S O_{q}(3)$-equivariantly Morita equivalent to $B$ since $V(1 / 2) \otimes V(1 / 2) \cong V(0) \oplus V(1)$ is an honest representation of $\mathrm{SO}_{q}(3)$.

The second part of the lemma follows from this. More precisely, the functor $F$ : $S O_{q}(3)$-Alg $\rightarrow K K^{S O_{q}(3)}$ given by $F(B)=\mathbb{K}(V(1 / 2)) \otimes B$ is homotopy invariant, stable, and split exact. By the universal property of equivariant $K K$-theory [20], it therefore induces a functor $f: K K^{S O_{q}(3)} \rightarrow K K^{S O_{q}(3)}$. Alternatively, one can also construct $f$ directly on the level of Kasparov cycles. Using the above Morita equivalence, one checks that $f^{2}$ is naturally isomorphic to the identity. In particular, $f$ is a natural isomorphism.

We need some basic calculations. Let us write

$$
A=M_{k_{1}}(\mathbb{C}) \oplus \cdots \oplus M_{k_{n}}(\mathbb{C})
$$

and view it as a $G$ - $C^{*}$-algebra with the defining action of $G=\operatorname{Qut}(A, \omega)$. Moreover we write write $\mathbb{C}$ for the trivial $G$ - $C^{*}$-algebra. As indicated above, we may identify $\operatorname{Irr}(G) \cong \mathbb{N}_{0}$ and $R(G)=\mathbb{Z}[t]$, where $t$ corresponds to the underlying representation of $A$, that is, $t=V(0)+V(1)$ under the identification with $R\left(S O_{q}(3)\right)=$ $K_{*}\left(C^{*}\left(S O_{q}(3)\right)\right)$. Similarly, we let $R^{\omega}(G)=K_{*}\left(C_{\omega}^{*}\left(S O_{q}(3)\right)\right)$, and identify $R^{\omega}(G)=$ $t^{1 / 2} \mathbb{Z}[t]$ as an $R(G)$-module. Here the generator $t^{1 / 2}$ corresponds to $V(1 / 2)$.

The representation ring $R(G)$ acts on the groups $K K^{G}(B, C)$ in a natural way, compare [15]. We have to identify these $R(G)$-modules in a few special cases.

Lemma 5.4. Let $G=\operatorname{Qut}(A, \omega)$ as above. Then we have isomorphisms

$$
\begin{aligned}
& K K^{G}\left(\mathbb{C}, C^{\mathrm{r}}(G)\right) \cong K K(\mathbb{C}, \mathbb{C})=\mathbb{Z} \\
& K K^{G}\left(A, C^{\mathrm{r}}(G)\right) \cong K K(A, \mathbb{C})=\mathbb{Z}^{n}
\end{aligned}
$$


and

$$
\begin{aligned}
K K^{G}(\mathbb{C}, \mathbb{C}) \cong R(G) & \cong K K^{G}(A, A) \\
K K^{G}(A, \mathbb{C}) \cong R^{\omega}(G) & \cong K K^{G}(\mathbb{C}, A)
\end{aligned}
$$

of $R(G)$-modules.

Proof. In the same way as in the proof of proposition 4.7 in [20] one checks the first two isomorphisms. Indeed, although the quantum group $G$ will typically fail to be coamenable, the arguments given there essentially carry over because $\breve{G}$ is $K$-amenable, see theorem 5.2 , The remaining isomorphisms follow from lemma 5.3 , The $R(G)$-module structures on the various Kasparov groups involving $A$ and $\mathbb{C}$ correspond to the obvious ones on $R(G)$ and $R^{\omega}(G)$.

We are now ready to prove our main theorem.

Theorem 5.5. Let

$$
A=M_{k_{1}}(\mathbb{C}) \oplus \cdots \oplus M_{k_{n}}(\mathbb{C})
$$

be a finite dimensional $C^{*}$-algebra of dimension at least 4 , equipped with a $\delta$-form $\omega$ for some $\delta>0$. Then

$$
K_{0}(C(G))=\mathbb{Z}^{(n-1)^{2}+1} \oplus \mathbb{Z}_{d}^{2 n-1}, \quad K_{1}(C(G))=\mathbb{Z},
$$

where $\mathbb{Z}_{d}$ is the finite cyclic group of order $d=\operatorname{gcd}\left(k_{1}, \ldots, k_{n}\right)$, the greatest common divisor of the numbers $k_{1}, \ldots, k_{n}$.

Proof. Notice that according to theorem 5.2 the dual of the quantum automorphism group $G=\operatorname{Qut}(A, \omega)$ is $K$-amenable, so that we do not have to distinguish between $C^{\mathrm{f}}(G)$ and $C^{\mathrm{r}}(G)$ as far as $K$-theory is concerned.

In order to compute the $K$-groups $K_{*}\left(C^{\mathrm{f}}(G)\right) \cong K_{*}\left(C^{\mathrm{r}}(G)\right)$ we have to write down a suitable resolution of the trivial action on $\mathbb{C}$ in $K K^{\check{G}}$. It is slightly easier to work in the compact picture, so that we shall construct a resolution of $C^{\mathrm{r}}(G)$ in $K K^{G}$. The corresponding homological algebra can be expressed using the framework of homological ideals, see [18. In order to do this it is convenient to first pass from $K K^{G}$ to $K K^{H}$ for $H=S O_{q}(3)$ monoidally equivalent to $G$. We define an ideal $\mathfrak{J}_{H} \subset K K^{H}$ by taking the intersection $\mathfrak{J}_{H}=\operatorname{ker}\left(F_{0}\right) \cap \operatorname{ker}\left(F_{1}\right)$ where $F_{j}: K K^{H} \rightarrow$ $K K$ are the functors given by

$$
F_{0}(C)=H \ltimes C, \quad F_{1}(C)=H \ltimes(\mathbb{K}(V(1 / 2)) \otimes C),
$$

respectively. It is straightforward to check that $\mathfrak{J}_{H}$ is a stable homological ideal in $K K^{H}$, and we let $\mathfrak{J}_{G}$ be the corresponding ideal in $K K^{G}$. The general machinery in [18], 15] now allows us to study $\mathfrak{J}_{G}$-projective resolutions in $K K^{G}$.

Recall from lemma 4.4 that the defining coaction on $A$ and the trivial coaction on $\mathbb{C}$ are the only torsion coactions of $\breve{G}$ up to equivariant Morita equivalence. It is straightforward to check that both these algebras are $\mathfrak{J}_{G}$-projective when viewed as objects in $K K^{G}$.

Let us consider the diagram $C$ • given by

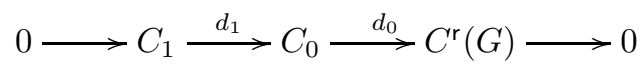

in $K K^{G}$ where

$$
\begin{aligned}
& C_{1}=\mathbb{C}^{\oplus n} \oplus A \\
& C_{0}=A^{\oplus n} \oplus \mathbb{C}
\end{aligned}
$$

and the arrows are defined as follows. The morphism $d_{0}$ is

$$
d_{0}=\epsilon_{1} \oplus \cdots \oplus \epsilon_{n} \oplus u
$$


where $\epsilon_{j}$ corresponds to the canonical basis vector $e_{j}$ in $\mathbb{Z}^{n}=K K^{G}\left(A, C^{r}(G)\right)$ and $u: \mathbb{C} \rightarrow C^{r}(G)$ is the unit homomorphism. The morphism $d_{1}$ is

$$
d_{1}=\left(\begin{array}{ccccc}
t^{1 / 2} & 0 & \cdots & & -k_{1} \\
0 & \ddots & & & \vdots \\
\vdots & & \ddots & & \\
& & & t^{1 / 2} & -k_{n} \\
-k_{1} & \cdots & & -k_{n} & t^{1 / 2}
\end{array}\right)
$$

where we use the identifications obtained in lemma 5.4.

In order to show that $C$ • is $\mathfrak{J}$-exact, it suffices to check that the sequences $K K^{G}\left(\mathbb{C}, C_{\bullet}\right)$ and $K K^{G}\left(A, C_{\bullet}\right)$ are both exact.

Let us compute the induced maps in the sequence $K K^{G}(\mathbb{C}, C \bullet)$. For $d_{0}$ we obtain

$$
d_{0}^{\mathbb{C}}=\left(\begin{array}{llll}
k_{1} & \cdots & k_{n} & 1
\end{array}\right),
$$

viewed as the $R(G)$-linear map acting on the free $R(G)$-module $K K^{G}\left(\mathbb{C}, C_{0}\right) \cong$ $R^{\omega}(G)^{\oplus n} \oplus R(G) \cong R(G)^{\oplus n+1}$. Indeed, the generator $t^{1 / 2}$ in the $j$-th copy of this module corresponds to the unit map $\mathbb{C} \rightarrow A$, and composing with $\epsilon_{j}$ yields $k_{j} \in \mathbb{Z}=K K^{G}\left(\mathbb{C}, C^{\mathrm{r}}(G)\right.$. Moreover, the generator 1 in the last copy of $R(G)$ is clearly mapped to $1 \in \mathbb{Z}$.

The morphism $d_{1}^{\mathbb{C}}$ becomes

$$
d_{1}^{\mathbb{C}}=\left(\begin{array}{ccccc}
1 & 0 & \ldots & & -k_{1} \\
0 & \ddots & & & \vdots \\
\vdots & & \ddots & & \\
& & & 1 & -k_{n} \\
-k_{1} & \cdots & & -k_{n} & t
\end{array}\right) .
$$

It is straightforward to check that $K K^{G}(\mathbb{C}, C \bullet)$ is an exact complex. Similarly, for the induced maps in $K K^{G}(A, C \bullet)$ we obtain

$$
d_{0}^{A}=\left(\begin{array}{cccccc}
1 & 0 & \ldots & & & k_{1} \\
0 & \ddots & & & & \vdots \\
\vdots & & \ddots & & & \vdots \\
& & & \ddots & & \vdots \\
0 & \ldots & & 0 & 1 & k_{n}
\end{array}\right)
$$

and

$$
d_{1}^{A}=\left(\begin{array}{ccccc}
t & 0 & \ldots & & -k_{1} \\
0 & \ddots & & & \vdots \\
\vdots & & \ddots & & \vdots \\
& & & t & -k_{n} \\
-k_{1} & \cdots & & -k_{n} & 1
\end{array}\right) .
$$

The resulting diagram is an exact complex as well, but this is slightly more subtle. Indeed, one has to be careful to take into account the correct $R(G)$-module structures when identifying the action of $t$. With this in mind, in order to check $\operatorname{ker}\left(d_{0}^{A}\right)=\operatorname{im}\left(d_{1}^{A}\right)$ one has to inductively reduce every element in $\operatorname{ker}\left(d_{0}^{A}\right)$ to an $n$ tuple of constant polynomials modulo $\operatorname{im}\left(d_{1}^{A}\right)$, and such elements are in the image of $d_{0}^{A}$. The surjectivity of $d_{0}^{A}$ and the injectivity of $d_{1}^{A}$ are easy.

It follows that $C \bullet$ is indeed a $\mathfrak{J}$-projective resolution, and to compute the $K$-theory 
of $C(G)$ it suffices to compute kernel and cokernel of the map $\partial: \mathbb{Z}^{n} \oplus \mathbb{Z}^{n}=$ $K_{0}\left(\mathbb{C}^{\oplus n} \oplus A\right) \rightarrow K_{0}\left(A^{\oplus n} \oplus \mathbb{C}\right)=\left(\mathbb{Z}^{n}\right)^{n} \oplus \mathbb{Z}$ given by

$$
\partial=\left(\begin{array}{ccccc}
\mathbf{k}^{T} & 0 & \ldots & & -k_{1} \mathbf{1} \\
0 & \ddots & & & \vdots \\
\vdots & & \ddots & & \vdots \\
& & & \mathbf{k}^{T} & -k_{n} \mathbf{1} \\
-k_{1} & \cdots & & -k_{n} & \mathbf{k}
\end{array}\right) .
$$

Here $\mathbf{k}^{T}$ is the transpose of $\left(k_{1}, \ldots, k_{n}\right)=\mathbf{k}$ and $\mathbf{1}$ is the identity matrix in $M_{n}(\mathbb{Z})$. Let us compute $\operatorname{ker}(\partial)$. Inspecting the $r n+1$ th rows of $\partial$ for $r=0, \ldots, n-1$ we see that an element of $\operatorname{ker}(\partial)$ is necessarily of the form $\left(a_{1}, \ldots, a_{n}, a_{1}, \ldots, a_{n}\right)$. Moreover, the first $n$ rows give the relations $k_{i} a_{1}=k_{1} a_{i}$, the next $n$ rows give $k_{i} a_{2}=k_{2} a_{i}$, and so on. That is, we obtain

$$
k_{i} a_{j}=k_{j} a_{i}
$$

for all $1 \leq i, j \leq n$. Notice in particular that $a_{2}, \ldots, a_{n}$ are uniquely determined by $a_{1} \in \mathbb{Z}$.

The general solution to these equations is $\left(a_{1}, \ldots, a_{n}\right)=\left(m k_{1} / d, \ldots, m k_{n} / d\right)$ where $m \in \mathbb{Z}$ and $d=\operatorname{gcd}\left(k_{1}, \ldots, k_{n}\right)$ is the greatest common divisor of $k_{1}, \ldots, k_{n}$. In particular, we conclude

$$
\operatorname{ker}(\partial)=\mathbb{Z}
$$

Let us now compute coker $(\partial)$. Using elementary row operations we can transform $\partial$ to

$$
\partial_{1}=\left(\begin{array}{ccccc}
\mathbf{k}^{T} & 0 & \ldots & & -k_{1} \mathbf{1} \\
0 & \ddots & & & \vdots \\
\vdots & & \ddots & & \vdots \\
& & & \mathbf{k}^{T} & -k_{n} \mathbf{1} \\
0 & \cdots & & 0 & \mathbf{0}
\end{array}\right)
$$

We thus obtain a direct summand $\mathbb{Z}$ in $\operatorname{coker}(\partial)$, and we may restrict to the matrix obtained by deleting the last row from $\partial_{1}$. Performing elementary row and column operations we may reduce the resulting matrix to

$$
\partial_{2}=\left(\begin{array}{ccccc}
\mathbf{v}^{T} & 0 & \ldots & & -k_{1} \mathbf{1} \\
0 & \ddots & & & \vdots \\
\vdots & & \ddots & & \vdots \\
& & & \mathbf{v}^{T} & -k_{n} \mathbf{1}
\end{array}\right)
$$

where $\mathbf{v}=(d, 0, \ldots, 0)$, and we recall that $d=\operatorname{gcd}\left(k_{1}, \ldots, k_{n}\right)$. Simplifying the right hand side of this matrix further leads to a diagonal matrix with $n+(n-1)$ entries $d$, and all remaing entries zero. Hence the final result is

$$
\operatorname{coker}(\partial)=\mathbb{Z}^{(n-1)^{2}+1} \oplus \mathbb{Z}_{d}^{2 n-1}
$$

as claimed.

Let us remark that the case $n=1$ of theorem 5.5 was already discussed in [28. At the opposite extreme $k_{1}=\cdots=k_{n}=1$, theorem 5.5 implies the following result.

Corollary 5.6. Let $n \geq 4$. Then the quantum permutation group $S_{n}^{+}$is $K$ amenable, and the $K$-theory is given by

$$
K_{0}\left(C\left(S_{n}^{+}\right)\right)=\mathbb{Z}^{n^{2}-2 n+2}, \quad K_{1}\left(C\left(S_{n}^{+}\right)\right)=\mathbb{Z} .
$$

Generators in degree zero are given by the projections 1 and $u_{i j}$ for $1 \leq i, j \leq n-1$. 
Proof. It remains only to verify the claim regarding generators of $K_{0}\left(C\left(S_{n}^{+}\right)\right)$. For this it suffices to consider the images of the generating projections $u_{i j} \in C^{\mathrm{f}}\left(S_{n}^{+}\right)$in $K_{0}\left(C\left(S_{n}\right)\right)$, and notice that they span a copy of $\mathbb{Z}^{n^{2}-2 n+2}$ inside $K_{0}\left(C\left(S_{n}\right)\right)=\mathbb{Z}^{n !}$. Essentially, in each row and column of the matrix $u=\left(u_{i j}\right)$ the last entry is determined by the remaining $n-1$ entries, with no further relations. This accounts for $(n-1)^{2}=n^{2}-2 n+1$ generators, and the missing generator is the class of the unit.

By mapping $C\left(S_{n}^{+}\right)$to $C\left(S_{4}^{+}\right)$and using theorem 5.2 in [6], it is not hard to check that the defining unitary $u \in M_{n}\left(C\left(S_{n}^{+}\right)\right)$yields a nonzero class $[u] \in K_{1}\left(C\left(S_{n}^{+}\right)\right)=$ $\mathbb{Z}$, and that $[u]$ is of the form $k x$ where $x$ is a generator and $k \in \mathbb{N}$ is at most 8 . However, to actually identify the generator of $K_{1}\left(C\left(S_{n}^{+}\right)\right)$would require more work. Let us point our that corollary 5.6 shows in particular that the $K$-theory of $C\left(S_{4}^{+}\right)=$ $C\left(S O_{-1}(3)\right)$ differs significantly from the $K$-theory of $S O(3)$.

Using the explicit structure of $C^{\mathrm{r}}\left(S_{n}^{+}\right)$for $n=1,2,3$ and corollary 5.6. we can distinguish the reduced $C^{*}$-algebras $C^{\mathrm{r}}\left(S_{n}^{+}\right)$for different values of $n$.

Corollary 5.7. Let $m, n \in \mathbb{N}$. Then $C^{\mathrm{r}}\left(S_{m}^{+}\right) \cong C^{\mathrm{r}}\left(S_{n}^{+}\right)$iff $m=n$.

Of course, this result holds for the maximal $C^{*}$-algebras as well. Notice that the maximal $C^{*}$-algebras can already be distinguished by comparing their abelianisations, a method which does not work for the reduced $C^{*}$-algebras.

\section{REFERENCES}

[1] Saad Baaj and Georges Skandalis. Unitaires multiplicatifs et dualité pour les produits croisés de $C^{*}$-algèbres. Ann. Sci. École Norm. Sup. (4), 26(4):425-488, 1993.

[2] Teodor Banica. Fusion rules for representations of compact quantum groups. Exposition. Math., 17(4):313-337, 1999.

[3] Teodor Banica. Symmetries of a generic coaction. Math. Ann., 314(4):763-780, 1999.

[4] Teodor Banica. Quantum groups and Fuss-Catalan algebras. Comm. Math. Phys., 226(1):221-232, 2002.

[5] Teodor Banica. Quantum automorphism groups of small metric spaces. Pacific J. Math., 219(1):27-51, 2005.

[6] Teodor Banica and Julien Bichon. Quantum groups acting on 4 points. J. Reine Angew. Math., 626:75-114, 2009.

[7] Teodor Banica, Julien Bichon, and Benoît Collins. Quantum permutation groups: a survey. In Noncommutative harmonic analysis with applications to probability, volume 78 of Banach Center Publ., pages 13-34. Polish Acad. Sci. Inst. Math., Warsaw, 2007.

[8] Teodor Banica, Stephen Curran, and Roland Speicher. De Finetti theorems for easy quantum groups. Ann. Probab., 40(1):401-435, 2012.

[9] Teodor Banica and Roland Speicher. Liberation of orthogonal Lie groups. Adv. Math., 222(4):1461-1501, 2009.

[10] Julien Bichon, An De Rijdt, and Stefaan Vaes. Ergodic coactions with large multiplicity and monoidal equivalence of quantum groups. Comm. Math. Phys., 262(3):703-728, 2006.

[11] Michael Brannan. Reduced operator algebras of trace-perserving quantum automorphism groups. Doc. Math., 18:1349-1402, 2013.

[12] Kenny De Commer. Galois objects for algebraic quantum groups. J. Algebra, 321(6):17461785,2009

[13] An De Rijdt and Nikolas Vander Vennet. Actions of monoidally equivalent compact quantum groups and applications to probabilistic boundaries. Ann. Inst. Fourier (Grenoble), 60(1):169-216, 2010.

[14] Johan Kustermans and Stefaan Vaes. Locally compact quantum groups. Ann. Sci. École Norm. Sup. (4), 33(6):837-934, 2000.

[15] Ralf Meyer. Homological algebra in bivariant $K$-theory and other triangulated categories. II. Tbil. Math. J., 1:165-210, 2008.

[16] Ralf Meyer and Ryszard Nest. The Baum-Connes conjecture via localisation of categories. Topology, 45(2):209-259, 2006.

[17] Ralf Meyer and Ryszard Nest. An analogue of the Baum-Connes isomorphism for coactions of compact groups. Math. Scand., 100(2):301-316, 2007. 
[18] Ralf Meyer and Ryszard Nest. Homological algebra in bivariant $K$-theory and other triangulated categories. I. In Triangulated categories, volume 375 of London Math. Soc. Lecture Note Ser., pages 236-289. Cambridge Univ. Press, Cambridge, 2010.

[19] Colin Mrozinski. Quantum automorphism groups and SO(3)-deformations. J. Pure Appl. Algebra, 219(1):1-32, 2015.

[20] Ryszard Nest and Christian Voigt. Equivariant Poincaré duality for quantum group actions. J. Funct. Anal., 258(5):1466-1503, 2010.

[21] P. Podleś. Quantum spheres. Lett. Math. Phys., 14(3):193-202, 1987.

[22] Piotr M. Soltan. Quantum SO(3) groups and quantum group actions on $M_{2}$. J. Noncommut. Geom., 4(1):1-28, 2010.

[23] Stefaan Vaes and Roland Vergnioux. The boundary of universal discrete quantum groups, exactness, and factoriality. Duke Math. J., 140(1):35-84, 2007.

[24] A. Van Daele. An algebraic framework for group duality. Adv. Math., 140(2):323-366, 1998.

[25] Roland Vergnioux. $K$-amenability for amalgamated free products of amenable discrete quantum groups. J. Funct. Anal., 212(1):206-221, 2004.

[26] Roland Vergnioux and Christian Voigt. The $K$-theory of free quantum groups. Math. Ann., 357(1):355-400, 2013.

[27] Christian Voigt. The Baum-Connes conjecture for free orthogonal quantum groups. Adv. Math., 227(5):1873-1913, 2011.

[28] Christian Voigt. Quantum $S U(2)$ and the Baum-Connes conjecture. In Operator algebras and quantum groups, volume 98 of Banach Center Publ., pages 417-432. Polish Acad. Sci. Inst. Math., Warsaw, 2012.

[29] Shuzhou Wang. Quantum symmetry groups of finite spaces. Comm. Math. Phys., 195(1):195211,1998

School of Mathematics and Statistics, University of Glasgow, 15 University GarDens, Glasgow G12 8QW, United Kingdom

E-mail address: christian.voigt@glasgow.ac.uk 